\title{
COMMUTING FUNCTIONS WITH NO COMMON FIXED POINT $\left({ }^{1}\right)$
}

\author{
BY \\ WILLIAM M. BOYCE
}

Introduction. Let $f$ and $g$ be continuous functions mapping the unit interval $I$ into itself which commute under functional composition, that is, $f(g(x))=g(f(x))$ for all $x$ in $I$. In 1954 Eldon Dyer asked whether $f$ and $g$ must always have a common fixed point, meaning a point $z$ in $I$ for which $f(z)=z=g(z)$. A. L. Shields posed the same question independently in 1955, as did Lester Dubins in 1956. The problem first appears in the literature in [15] as part of a more general question raised by $J$. R. Isbell. The purpose of this paper is to answer Dyer's question in the negative by the construction of a pair of commuting functions which have no fixed point in common.

The connection between functions commuting and sharing fixed points appears in several areas of analysis. Perhaps the best-known example is the Markov-Kakutani theorem [11, p. 456], which states that a commuting family of continuous linear mappings of a compact convex subset of a linear topological space into itself has a common fixed point.

The earliest relevant work on commuting functions was done in the 1920's by J. F. Ritt, who published several papers in which he investigated the algebraic properties of functional composition as a binary operation on the set of rational complex functions. His most important result from the modern standpoint was a characterization of commuting (or permutable) rational functions [19]. He proved that if $f$ and $g$ are commuting polynomials, then, within certain homeomorphisms, either they are iterates of the same function $\left(f=F^{n}\right.$ and $g=F^{m}$ for some $\left.F, n, m\right)$, both powers of $x$, or both must be Tchebycheff polynomials (defined by the relationship $\left.T_{n}(\cos x)=\cos n x\right)$. In either case a common fixed point may be shown to exist, so commuting polynomials have a common fixed point.

The subject of commuting functions lay largely dormant until a 1951 paper by Block and Thielman [6] presented some new results on families of commuting polynomials and called attention to Ritt's earlier work. Their paper, together with the connection between commutativity and common fixed points found in other areas of mathematics, seems to have been the inspiration for the questions cited above.

In the last few years a number of papers have been published on commuting

Received by the editors January 9, 1967 and, in revised form, December 1, 1967.

( ${ }^{1}$ ) This paper is a condensation of the author's 1967 doctoral dissertation at Tulane University under Professor G. S. Young. 
functions, all of them apparently motivated by the conjecture of a common fixed point. These are cited in the list of references. Although the basic question seems topological, the methods employed on the subject have included almost everything but topology, from complex variables to matrices. Some papers have treated related problems, while others have attacked the problem directly but for special cases.

The generation of this counterexample was the result of an extensive investigation of the connection between commuting functions and the permutations defined by Baxter [1] and Baxter and Joichi [3]. These permutations prescribe the manner in which $f$ and $g$ act on the fixed-point sets of $f g$ and $g f$. The author investigated pairs of functions for which the number $N$ of Type I and II fixed points [1] varied between five and thirteen ( $N$ is odd by definition). For $N$ up through nine the conjecture is true, but for three of the cases with $N=11$ the issue is still in doubt. (In unpublished work [2], Baxter and Joichi have also worked through nine and met a "stumbling block" at eleven.) The three unresolved cases are represented by the following permutations:

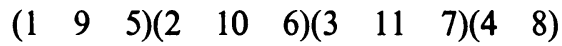

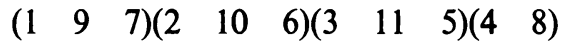

$$
\begin{aligned}
& \left(\begin{array}{lllllll}
1 & 11 & 9 & 5 & 3 & 7
\end{array}\right)\left(\begin{array}{lllll}
2 & 10 & 8 & 4 & 6
\end{array}\right) \text {. }
\end{aligned}
$$

The principal difficulty in all these cases is in getting the approximating functions to converge uniformly.

The particular example presented in this paper was developed from a case with $N=13$, represented by the permutation

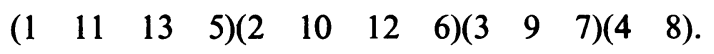

The author used a digital computer to generate the " $w$-admissible" permutations [3], screen out those with fixed points, and divide them into equivalence classes for further investigation. The theorems and algorithms used are described in [7]. Even after this automatic processing there were 22 cases for $N=11$; for $N=13$ there were 112 , of which the counterexample was number 101 . The other 111 cases for $N=13$ either must have a common fixed point or cannot be induced by commuting functions.

Independent of and contemporary with the author's work, J. P. Huneke has also obtained examples of pairs of commuting functions with no common fixed point [13], [14]. One of Huneke's examples is identical to the one presented here.

Construction of the functions. The desired functions $f$ and $g$ will be constructed as the limits of a pair of sequences of continuous functions

$$
f_{1}, f_{2}, f_{3}, \ldots \rightarrow f, \quad g_{1}, g_{2}, g_{3}, \ldots \rightarrow g .
$$

The construction associates with each pair of functions $\left\{f_{i}, g_{i}\right\}$ a set of "stable points" $S_{i}$ such that $S_{i} \subset S_{i+1}$, and for $x$ in $S_{i}, f_{i+j}(x)=f_{i}(x)$ and $g_{i+j}(x)=g_{i}(x)$ for 
all $j \geqq 0$. Thus in the limit $f(x)=f_{i}(x)$ and $g(x)=g_{i}(x)$ for $x$ in $S_{i}$. The union of the $S_{i}$ is dense in $I$, so the limit functions $f$ and $g$ will be determined by the values of $f_{i}$ and $g_{i}$ on $S_{i}$ and be independent of the values on the " $(i)$-intervals" in between.

In the construction only the first two pairs of functions with their stable points are explicitly stated; the rest of the functions in each sequence are defined inductively. Graphs of the first four functions in each sequence are shown in Figures $2,3,4$, and 5 . Note that from the first three pairs of functions one could easily infer that as $i$ increases the differences between successive functions will be limited to ever-smaller intervals in the center of $I$. However, using $f_{4}$ and $g_{4}$ one obtains the correct impression, namely, that with increasing $i$ the differences tend to spread out over more and more of the interval.

The steps of the construction are as follows: first, necessary definitions are stated and the initial functions specified; then a lemma is given which produces the sequences of functions; and finally, a theorem is proven which derives the desired properties of the limit functions.

Definition. Let $A$ be a closed interval. A subset $T$ of $A$ is the (k)-set of $A$ if it has $k$ elements, contains the end points of $A$, and divides $A$ into $k-1$ subintervals of equal lengths.

Definition. Let $A$ and $B$ be closed intervals; let $T$ be the $(2 k+2)$-set of $A$ and $U$ be the (2k)-set of $B$ for $k \geqq 2$. Let $T=\left\{t_{1}, t_{2}, \ldots, t_{2 k+2}\right\}$ and $U=\left\{u_{1}, u_{2}, \ldots, u_{2 k}\right\}$, where the elements of $T$ and $U$ are numbered in increasing order. Four functions from $A$ onto $B$ will be called the $(2 k+2)$-hook functions from $A$ onto $B$. Examples of these are given in Figure 1. The order of each of the four functions is $2 k+2$. Each has a direction-increasing or decreasing-and a type-maximum or minimum. Each will map $T$ onto $U$ and be linear on the intervals between points of $T$; thus it suffices to define them explicitly only on $T$, as follows:

\begin{tabular}{|c|c|c|c|c|c|}
\hline Type & Direction & \multicolumn{4}{|c|}{ Value of the function at $t_{i}$} \\
\hline Minimum & Increasing & $u_{i}$, & $i \leqq 2$ & $u_{i-2}$ & $i>2$ \\
\hline Maximum & Increasing & $u_{i}$ & $i \leqq 2 k$ & $u_{i-2}$ & $i>2 k$ \\
\hline Minimum & Decreasing & $u_{2 k+1-i}$ & $i \leqq 2 k$ & $u_{2 k+3-i}$ & $i>2 k$ \\
\hline Maximum & Decreasing & $u_{2 k+1-i}$ & $i \leqq 2$ & $u_{2 k+3-i}$ & $i>2$ \\
\hline
\end{tabular}

The terms $(2 k+2)$-hook function or simply hook function may be used where the order or intervals involved are understood.

Definition. Given a finite subset $S_{i}$ of $I$, a nondegenerate closed interval $J \subset I$ is an (i)-interval if its end points are in $S_{i}$ but none of its interior points are.

Definition. The mesh size $M_{i}$ of $S_{i}$ is the length of the longest (i)-interval.

Next the initial functions with their associated sets of stable points will be specified. We will define $f_{i}, g_{i}$, and $S_{i}$ for $i=1$ and 2 . In each case $f_{i}$ and $g_{i}$ will be continuous and linear on each $(i)$-interval, so they may be defined by specifying only their values at the points of $S_{i}$. 

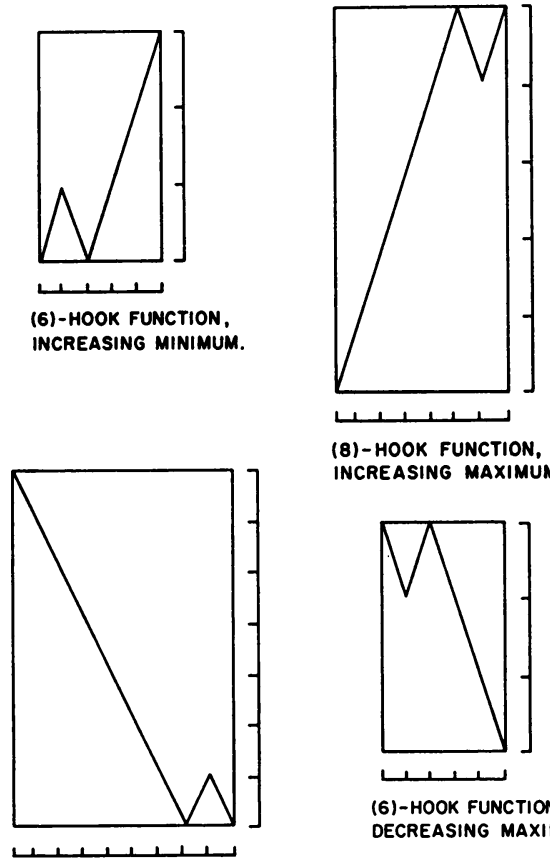
INCREASING MAXIMUM.

(10)- HOOK FUNCTION, DECREASING MINIMUM.

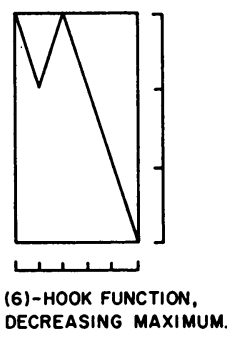

FIGURE 1

First we shall define $S_{1}=\{0,1 / 3,2 / 3,1\}$. Note that $S_{1}$ is the (4)-set of $I$ and that $M_{1}=1 / 3=(1 / 3)^{1}$. On $S_{1}$ the values of $f_{1}$ and $g_{1}$ are as follows:

\begin{tabular}{lcccc}
$S_{1}$ & 0 & $1 / 3$ & $2 / 3$ & 1 \\
\cline { 2 - 4 }$f_{1}$ & 1 & 0 & 1 & 0 \\
$g_{1}$ & 0 & 1 & 0 & 1
\end{tabular}

$f_{1}$ and $g_{1}$ are defined to be linear between points of $S_{1}$. Their graphs are given in Figure 2. $f_{1}$ and $g_{1}$ can be seen to commute and have a common fixed point, but no other pair of functions will have either of these properties.

To define $S_{2}$ we divide the first and third (1)-intervals into three subintervals, and the middle (1)-interval into five subintervals, so we have

$$
S_{2}=\{0,1 / 9,2 / 9,1 / 3,6 / 15,7 / 15,8 / 15,9 / 15,2 / 3,7 / 9,8 / 9,1\} .
$$

Observe that $S_{1} \subset S_{2}$ and $M_{2}=1 / 9=(1 / 3)^{2}$. Now $f_{2}$ and $g_{2}$ shall be defined on $S_{2}$ :

\begin{tabular}{lcccccccccccc}
$S_{2}$ & 0 & $1 / 9$ & $2 / 9$ & $1 / 3$ & $6 / 15$ & $7 / 15$ & $8 / 15$ & $9 / 15$ & $2 / 3$ & $7 / 9$ & $8 / 9$ & 1 \\
\cline { 2 - 10 }$f_{2}$ & 1 & $2 / 3$ & $1 / 3$ & 0 & $1 / 3$ & 0 & $1 / 3$ & $2 / 3$ & 1 & $2 / 3$ & $1 / 3$ & 0 \\
$g_{2}$ & 0 & $1 / 3$ & $2 / 3$ & 1 & $2 / 3$ & $1 / 3$ & 0 & $1 / 3$ & 0 & $1 / 3$ & $2 / 3$ & 1
\end{tabular}

$f_{2}$ and $g_{2}$ are linear between points of $S_{2}$. Their graphs are shown in Figure 3. 

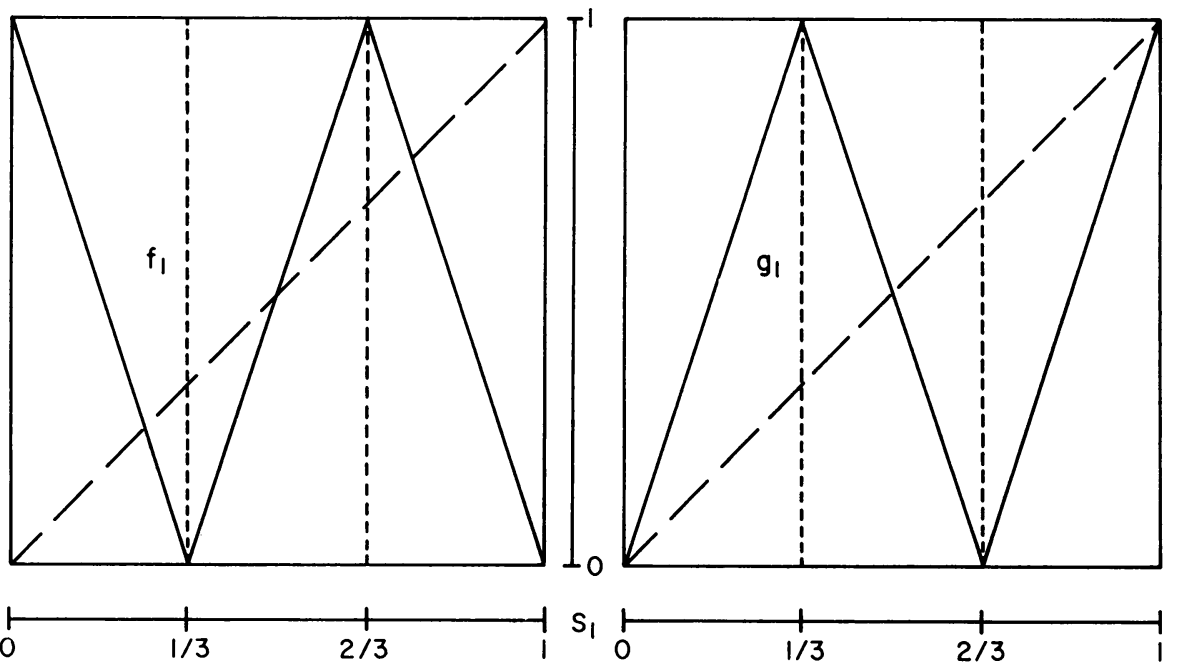

FIGURE 2

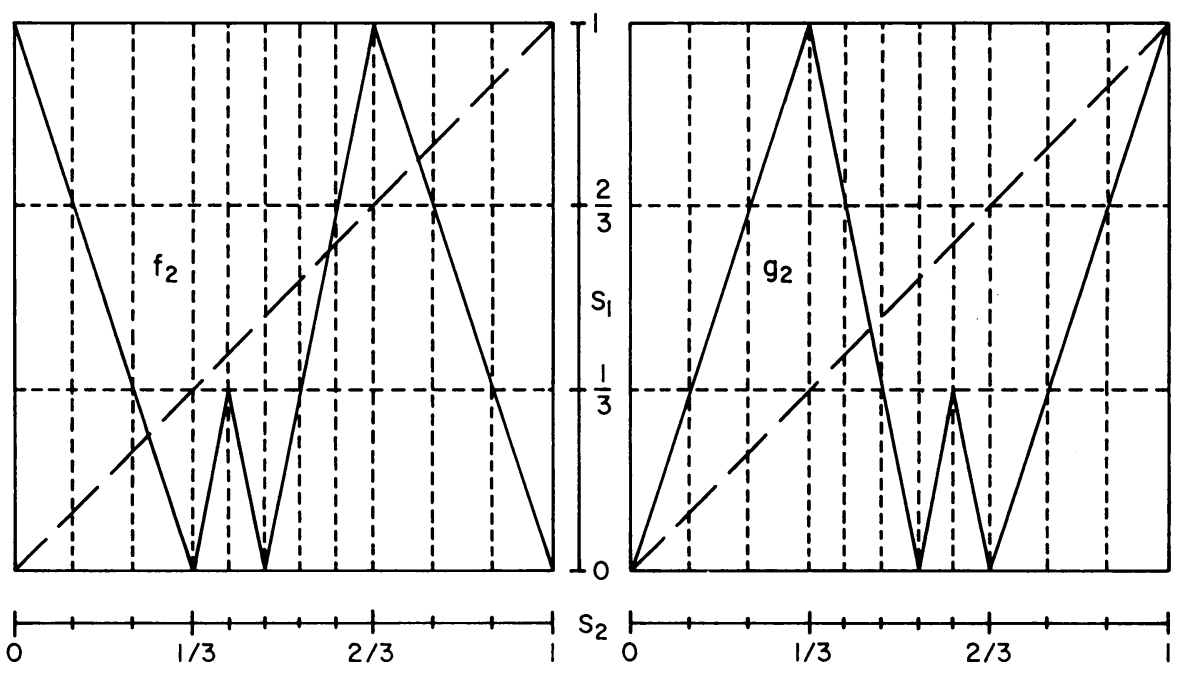

Figure 3

Note that $f_{2}\left(S_{2}\right)=S_{1}=g_{2}\left(S_{2}\right)$ and $f_{2}$ and $g_{2}$ are (6)-hook functions on the middle (1)-interval.

Figure 4 gives the graphs of $f_{3}$ and $g_{3}$ and Figure 5 the graphs of $f_{4}$ and $g_{4}$. These functions are constructed through the inductive process described below.

LemMa. There exist two sequences of functions $\left\{f_{i}\right\}$ and $\left\{g_{i}\right\}$ and a sequence of sets $\left\{S_{i}\right\}$ which satisfy the following requirements for $i \geqq 2$ :

(1) $f_{1}, f_{2}, g_{1}, g_{2}, S_{1}, S_{2}$ are as defined above.

(2) [properties of $S_{i}$ ] $S_{i-1} \subset S_{i}$; if J is an (i-1)-interval, then there is a $k \geqq 2$ such that $S_{i} \cap J$ is the $(2 k)$-set of $J$; and $M_{i} \leqq(1 / 3)^{i}$. 

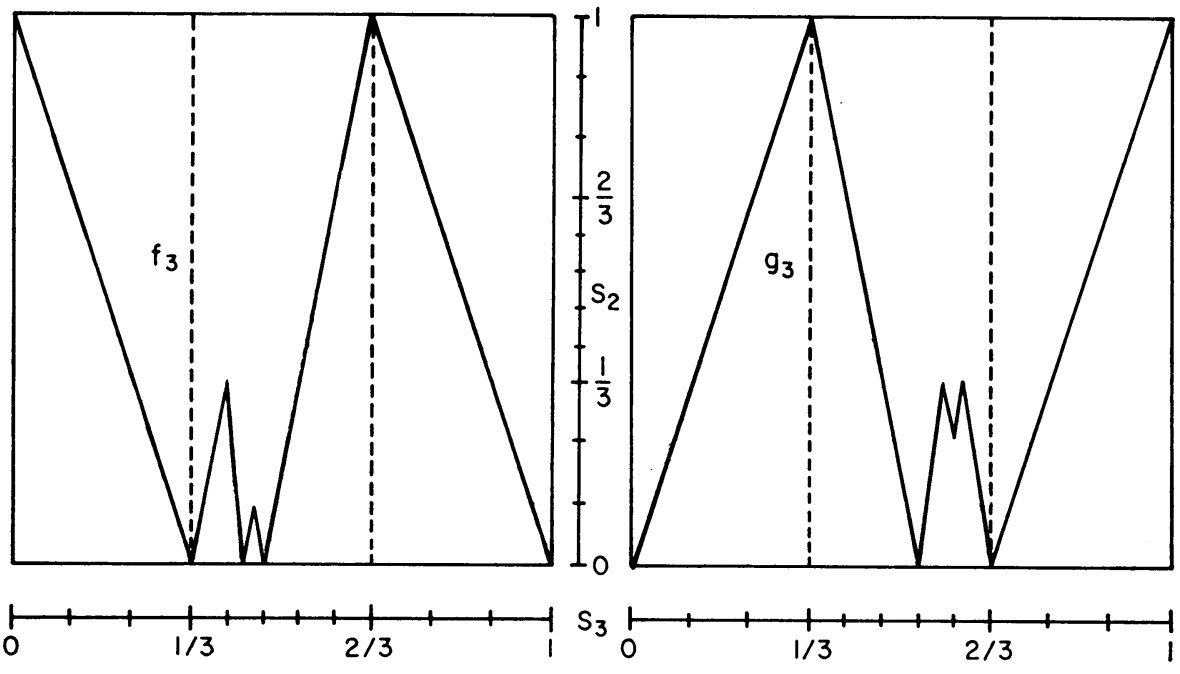

FIGURE 4
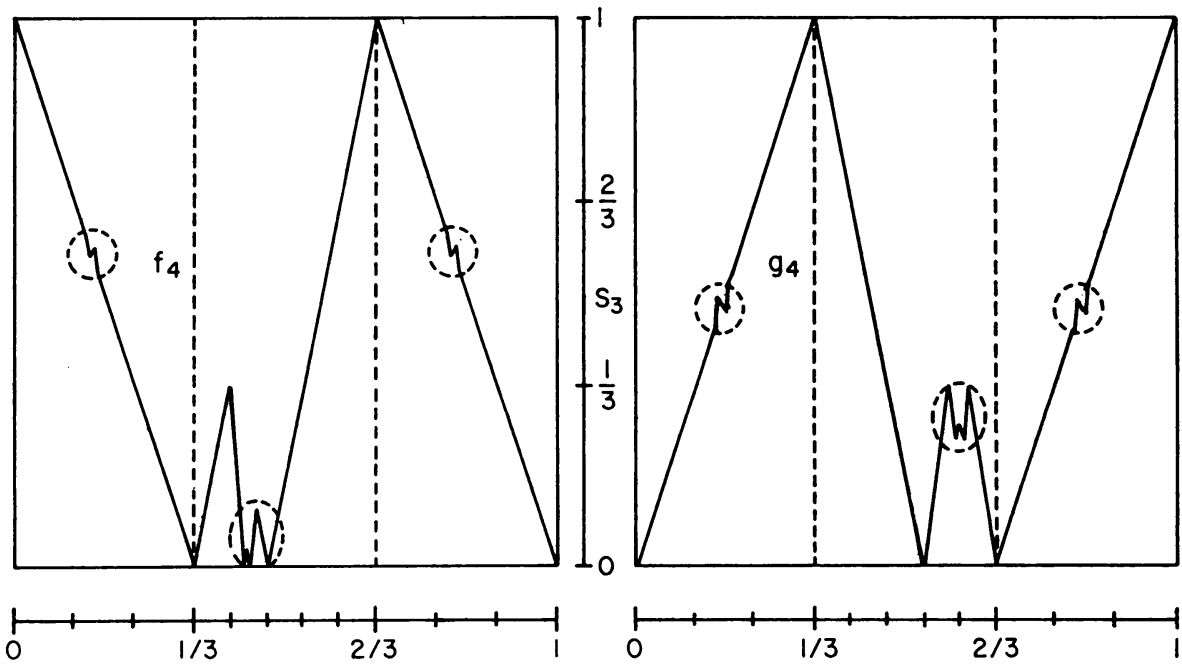

Figure 5

(3) [diagram commutativity] $f_{i-1} g_{i}=g_{i-1} f_{i}$ on $I$.

(4) [ $f_{i}$ on an (i)-interval] $f_{i}\left(S_{i}\right) \subset S_{i-1} ; f_{i}$ is linear on each (i)-interval and maps it onto an (i-1)-interval. Further, for $j \geqq 0, f_{i+j}$ agrees with $f_{i}$ on $S_{i}$, and if $J$ is an (i)-interval, then $f_{i+j}(J)=f_{i}(J)$; thus $\left\|f_{i+j}-f_{i}\right\| \leqq M_{i-1}$, where $\|F\|$ is the sup norm of $F$ on $I$.

(5) $\left[g_{i}\right.$ on an (i)-interval] same as 4 , except for $g_{i+j}$ and $g_{i}$.

(6) $\left[f_{i}\right.$ on an $(i-1)$-interval $]$ let $J$ be an $(i-1)$-interval and define $J_{f}=f_{i-1}(J)$. Then either

(a) $f_{i}$ is linear on $J, f_{i} / J=f_{i-1} / J$, and $S_{i} \cap J$ and $S_{i-1} \cap J_{f}$ have the same cardinality; or 
(b) $f_{i}$ is a $(2 k+2)$-hook function from $J$ onto $J_{f}, S_{i} \cap J$ is the $(2 k+2)$-set of $J$, and $S_{i-1} \cap J_{f}$ is the $(2 k)$-set of $J_{f}$.

(7) $\left[g_{i}\right.$ on an $(i-1)$-interval] same as 6 , except for $g_{i}, g_{i-1}$, and $J_{g}$.

Proof. The proof will be by induction. Let $L(n)$ designate the proposition that the functions $f_{i}$ and $g_{i}$ and sets $S_{i}$ are well defined for $1 \leqq i \leqq n$ and satisfy requirements $1-7$ of the lemma when $2 \leqq i \leqq n$ and $i+j \leqq n$. The proof of the lemma consists of first showing that $L(2)$ is true, then defining a construction by which $L(n)$ implies $L(n+1)$.

To prove $L(2)$, we first observe that requirement 1 only specifies the functions and sets we must consider. For requirement 2, we have earlier noted that $S_{1} \subset S_{2}$ and that $M_{2}=1 / 9=(1 / 3)^{2} . S_{2}$ divides the three (1)-intervals evenly into three, five, and three subintervals, respectively, so that the corresponding values of $k$ are two, three, and two. Let us momentarily defer requirement 3, the "diagram commutativity". For requirements 4 and 5 we have noted that $f_{2}\left(S_{2}\right)=S_{1}=g_{2}\left(S_{2}\right)$, so containment is implied. By definition, $f_{2}$ and $g_{2}$ are linear on (2)-intervals, and from the definitions we see that consecutive points of $S_{2}$ are mapped onto consecutive points of $S_{1}$ by both $f_{2}$ and $g_{2}$; thus the image of a (2)-interval is a single (1)-interval. The rest of 4 and 5 is satisfied trivially, since for $L(2)$ we need only consider $i+j$ $=2=i$. Now we may return to " diagram commutativity". Since $f_{2}$ and $g_{2}$ are linear on (2)-intervals and map (2)-intervals onto (1)-intervals, and $f_{1}$ and $g_{1}$ are linear on (1)intervals, it follows that $f_{1} g_{2}$ and $g_{1} f_{2}$ are linear on (2)-intervals. Thus to prove that the two composites agree on $I$ it suffices to show that they agree on $S_{2}$. Their values on $S_{2}$ can be computed easily from the definitions and are found to agree, as follows:

\begin{tabular}{ccccccccccccc}
$S_{2}$ & 0 & $1 / 9$ & $2 / 9$ & $1 / 3$ & $6 / 15$ & $7 / 15$ & $8 / 15$ & $9 / 15$ & $2 / 3$ & $7 / 9$ & $8 / 9$ & 1 \\
\cline { 2 - 10 }$g_{1} g_{2}=g_{1} f_{2}$ & 1 & 0 & 1 & 0 & 1 & 0 & 1 & 0 & 1 & 0 & 1 & 0
\end{tabular}

For requirements 6 and 7 we note that $J_{f}=I=J_{g}$ for all three (1)-intervals. For the first and third (1)-intervals we can see from Figure 3 that case (a) applies, since on both intervals $f_{2}$ and $g_{2}$ are linear. The cardinalities of $S_{2} \cap J$ and $S_{1} \cap I$ are both four. On the middle (1)-interval both $f_{2}$ and $g_{2}$ are (6)-hook functions from $J$ onto $I$, so that $k=2$. $f_{2} / J$ is minimum increasing and $g_{2} / J$ is minimum decreasing. $S_{2} \cap J$ is the (6)-set of $J$, dividing the interval into five subintervals, and $S_{1} \cap I$ is the (4)-set of $I$. Thus requirements $1-7$ are all met when $i=i+j=2$, and $L(2)$ is true.

Now we must devise a construction by which $L(n)$ will imply $L(n+1)$. We will obtain the functions and sets needed for $L(n+1)$ by simply adjoining $f_{n+1}, g_{n+1}$, and $S_{n+1}$ to the partial sequences of $f_{i}, g_{i}$, and $S_{i}$ for $i \leqq n$ which are assumed to exist as part of $L(n)$. The functions and set for $n+1$ must be defined in such a way as to satisfy requirements $1-7$ of the lemma when $i=n+1$ and $i+j=n+1$.

The need to satisfy the requirements goes a long way toward defining $f_{n+1}$, $g_{n+1}$, and $S_{n+1}$. For requirement 2 we must have $S_{n} \subset S_{n+1}$, and for requirements 4 and $5 f_{n+1}$ must agree with $f_{n}$ and $g_{n+1}$ agree with $g_{n}$ on $S_{n}$. Let $J$ be an $(n)$-interval 
and define $J_{f}=f_{n}(J)$ and $J_{g}=g_{n}(J)$. We know from requirements 4 and 5 of $L(n)$ that $J_{f}$ and $J_{g}$ are $(n-1)$-intervals, and in order to satisfy these same requirements for $L(n+1)$ we must define $f_{n+1}$ and $g_{n+1}$ so that $f_{n+1}(J)=J_{f}$ and $g_{n+1}(J)=J_{g}$. Since from requirements 4 and $5 f_{n}$ and $g_{n}$ are linear on $J$, we have the situation depicted in Figure 6. $f_{n+1}$ must agree with $f_{n}$ on the end points of $J$, since the end points are
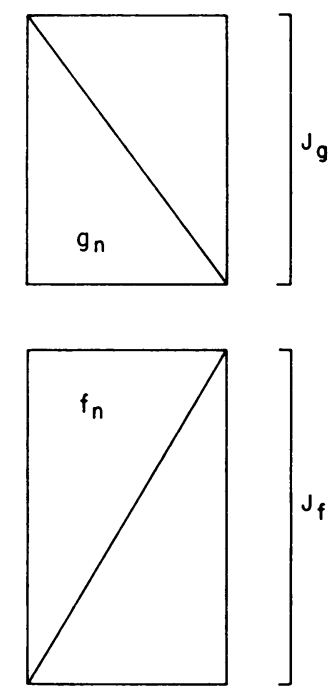

Figure 6

in $S_{n}$, and the image of $J$ under $f_{n+1}$ must be the same as its image under $f_{n}$. Thus the problem of defining $f_{n+1}$ on $J$ is that of deforming the graph of $f_{n}$ (if necessary) to get the graph of $f_{n+1}$ while staying within $J \times J_{f}$ and holding the values at the end points fixed. Similarly, we must define $g_{n+1}$ on $J$ so that the graph of $g_{n+1}$ remains in $J \times J_{g}$ and agrees with $g_{n}$ at the end points.

The need to satisfy requirement $3, f_{n} g_{n+1}=g_{n} f_{n+1}$, compels us to consider $f_{n}$ on $J_{g}$ and $g_{n}$ on $J_{f}$. We have previously noted that $J_{f}$ and $J_{g}$ are $(n-1)$-intervals. Using requirements 4 and 5 from $L(n)$ with $i=n-1$, we see that $g_{n-1}$ maps $J_{f}$ onto an $(n-2)$-interval $\left(J_{f}\right)_{g}$, which we will call $J_{h}$, and $f_{n-1}$ maps $J_{g}$ onto an $(n-2)$-interval $\left(J_{g}\right)_{f}$. But from the "commutativity" of $L(n)$ we know that $f_{n-1} g_{n}=g_{n-1} f_{n}$, so $\left(J_{g}\right)_{f}=f_{n-1}\left(J_{g}\right)=f_{n-1} g_{n}(J)=g_{n-1} f_{n}(J)=g_{n-1}\left(J_{f}\right)=\left(J_{f}\right)_{g}=J_{n}$. Also, since $n \geqq n-1$ and $J_{f}$ and $J_{g}$ are $(n-1)$-intervals, we can use 4 and 5 again to obtain $f_{n}\left(J_{g}\right)=f_{n-1}\left(J_{g}\right)=J_{n}$ and $g_{n}\left(J_{f}\right)=g_{n-1}\left(J_{f}\right)=J_{h}$. Thus we have the diagram in Figure 7 , with all functions onto. Furthermore, by 4 and 5 , if $x$ is one of the end points of $J$ then $f_{n}(x)$ and $g_{n}(x)$ are in $S_{n-1}$; but $f_{n}$ and $g_{n}$ agree with $f_{n-1}$ and $g_{n-1}$ on $S_{n-1}$, so $f_{n} g_{n}(x)=f_{n-1} g_{n}(x)$ $=g_{n-1} f_{n}(x)=g_{n} f_{n}(x)$. Thus $f_{n} g_{n}$ and $g_{n} f_{n}$ agree on the end points of $J$.

Let us now describe how to define $f_{n+1}, g_{n+1}$, and $S_{n+1}$. Since $I$ is covered by 


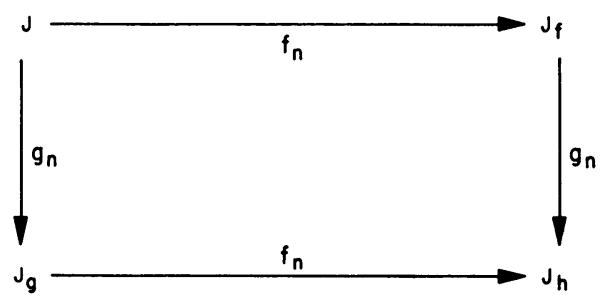

Figure 7

(n)-intervals, and we know that $f_{n+1}$ must agree with $f_{n}$ and $g_{n+1}$ agree with $g_{n}$ on $S_{n}$, the end points of the (n)-intervals, it suffices to give a construction for $f_{n+1}, g_{n+1}$, and $S_{n+1}$ only on the arbitrary ( $n$ )-interval $J$. So the problem reduces to (a) finding functions $f_{n+1}$ and $g_{n+1}$ to make the diagram in Figure 8 commute while $f_{n+1}$ and

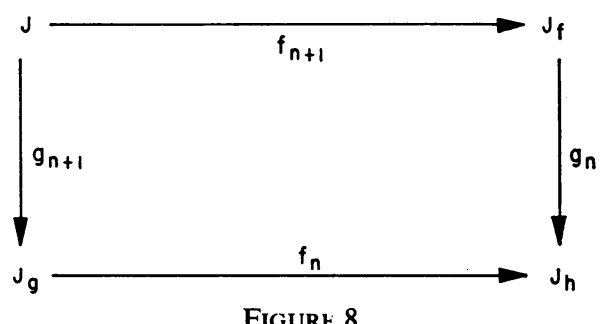

$g_{n+1}$ agree on the end points of $J$ with $f_{n}$ and $g_{n}$ and (b) defining $S_{n+1} \cap J$; so all meet requirements $1-7$ of $L(n+1)$.

Since $J_{f}$ and $J_{g}$ are $(n-1)$-intervals, we may apply requirements 6 and 7 of $L(n)$ to get information on $f_{n} / J_{g}, g_{n} / J_{f}, S_{n} \cap J_{f}$, and $S_{n} \cap J_{g}$ which will aid the construction. $J_{h}$ is an (n-2)-interval, so from requirement 2 we know that there is a $k \geqq 2$ such that $S_{n-1} \cap J_{h}$ is the ( $\left.2 k\right)$-set of $J_{h}$. Then from 6 we know that either (a) $f_{n}$ is linear on $J_{g}$ and $S_{n} \cap J_{g}$ is the (2k)-set of $J_{g}$; or (b) $f_{n}$ is a $(2 k+2)$-hook function from $J_{g}$ onto $J_{h}$ and $S_{n} \cap J_{g}$ is the $(2 k+2)$-set of $J_{g}$. A similar conclusion for $g_{n} / J_{f}$ and $S_{n} \cap J_{f}$ follows from requirement 7 . Note in particular that if $f_{n} / J_{g}$ and $g_{n} / J_{f}$ are both hook functions, then they have the same order, $2 k+2$.

If we now let functions be graphed "sideways", with the axis of the independent variable vertical and the dependent variable's axis horizontal, we can represent a typical situation as in Figure 9. In this figure $g_{n} / J_{f}$ and $f_{n} / J_{g}$ have been graphed sideways, and both are hook functions. The length of $J$ is somewhat exaggerated; $J$ and the subintervals of $J_{f}$ and $J_{g}$ are all (n)-intervals.

The problem divides naturally into four cases, depending on the properties of the functions $f_{n} / J_{g}$ and $g_{n} / J_{f}$ :

(I) Both functions are linear.

(II) One function is linear, the other is a hook function.

(III) Both are hook functions of the same type.

(IV) Both are hook functions, but of opposite type.

Recalling that $k$ is such that $S_{n-1} \cap J_{h}$ is the (2k)-set of $J_{h}$, for each of the four cases 

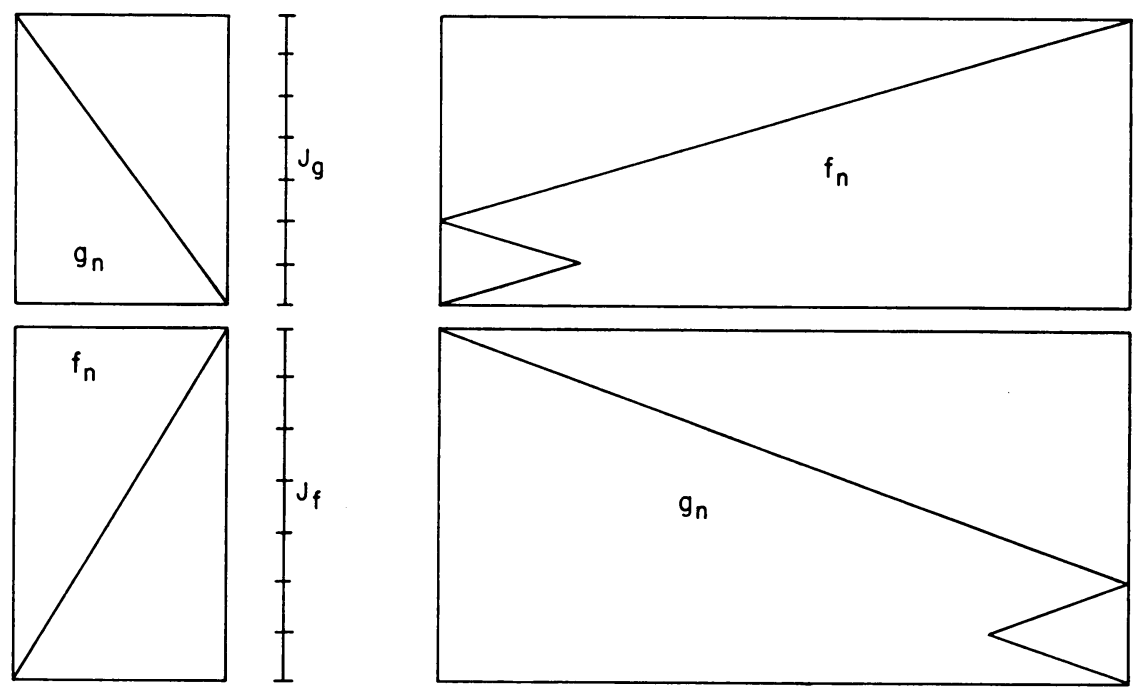

FIGURE 9

we define $f_{n+1}, g_{n+1}$, and $S_{n+1}$ as follows:

(I) Define $f_{n+1}=f_{n}$ and $g_{n+1}=g_{n}$ on $J$, and define $S_{n+1} \cap J$ to be the (2k)-set of $J$.

(II) Define $S_{n+1} \cap J$ to be the $(2 k+2)$-set of $J$. If $f_{n} / J_{g}$ is linear, then $f_{n+1}=f_{n}$ on $J$ and $g_{n+1}$ is a $(2 k+2)$-hook function from $J$ onto $J_{g}$ with the same direction as $g_{n} / J ; g_{n+1} / J$ has the same type as $g_{n} / J_{f}$ if and only if $f_{n} / J_{g}$ is increasing. (See Figure 10.) If $g_{n} / J_{f}$ is linear, then the same definition holds except with $f$ and $g$ reversed.

(III) Define $f_{n+1}=f_{n}$ and $g_{n+1}=g_{n}$ on $J$, and define $S_{n+1} \cap J$ to be the $(2 k+2)$ set of $J$.

(IV) Define $S_{n+1} \cap J$ to be the $(2 k+4)$-set of $J . f_{n+1} / J$ is a $(2 k+4)$-hook function with the same direction as $f_{n} / J$, and $\dot{g}_{n+1} / J$ is a $(2 k+4)$-hook function with the same direction as $g_{n} / J . f_{n+1} / J$ has the same type as $f_{n} / J_{g}$ if and only if $g_{n} / J_{f}$ is increasing, and $g_{n+1} / J$ has the same type as $g_{n} / J_{f}$ if and only if $f_{n} / J_{g}$ is increasing. (See Figure 11.)

Now it must be shown that requirements $1-7$ are satisfied by $f_{n+1}, g_{n+1}$, and $S_{n+1}$ as defined. Requirement 1 is inherited from $L(n)$. For requirement 2 , in each case $S_{n+1} \cap J$ is the $(2 m)$-set of $J$ for some $m$, so $S_{n} \subset S_{n+1}$. Since in each case the cardinality of $S_{n+1} \cap J$ is no less than that of $S_{n} \cap J_{f}$, which is at least four by $L(n)$, we must have $m \geqq 2$. Thus each $(n)$-interval is split into at least three equal subintervals by $S_{n+1}$, so we have $M_{n+1} \leqq(1 / 3) M_{n} \leqq(1 / 3)^{n+1}$.

Requirements 6 and 7 will be considered next. In case $I, f_{n} / J_{g}$ and $g_{n} / J_{f}$ are linear and so $S_{n} \cap J_{f}$ and $S_{n} \cap J_{g}$ are both (2k)-sets. We defined $S_{n+1} \cap J$ to be the 

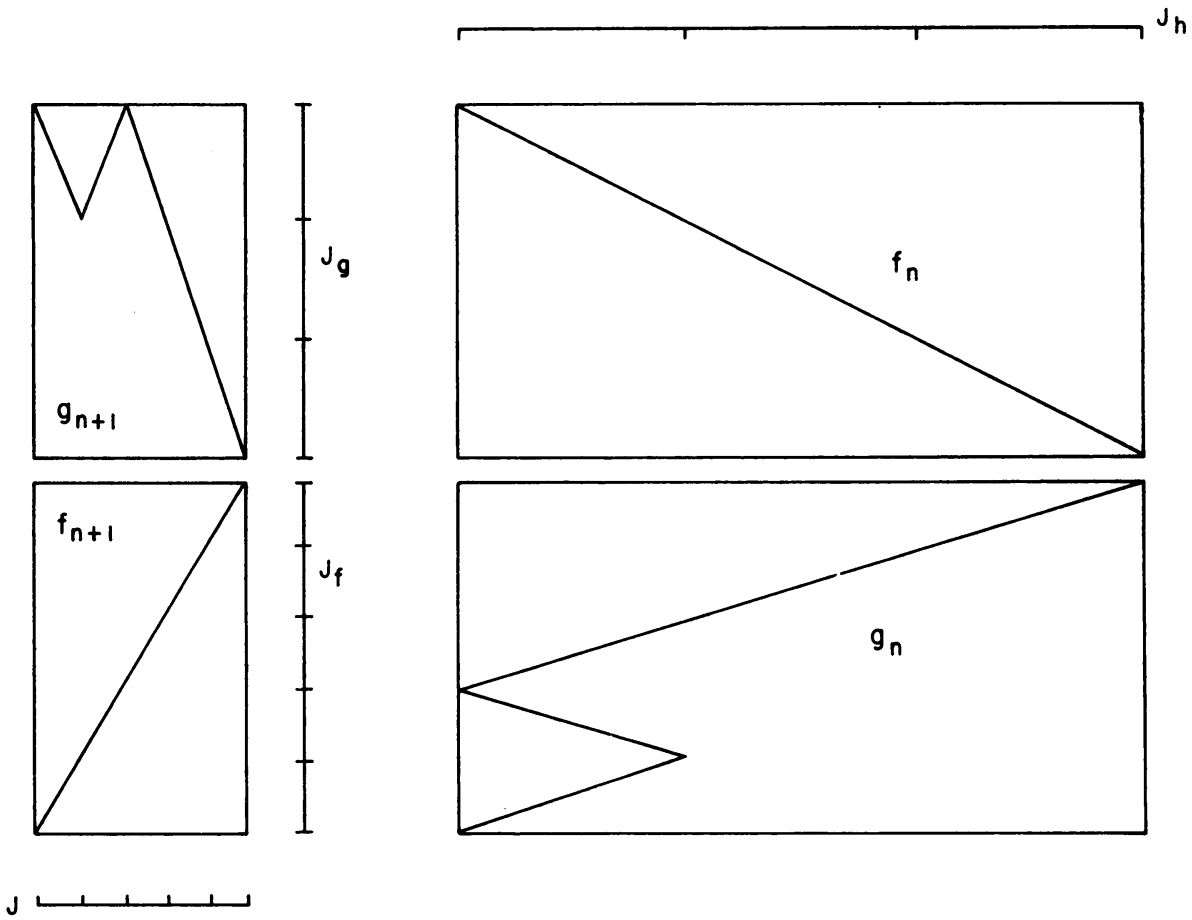

FIGURE 10

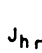

$$
\begin{aligned}
& f_{n} g_{n+1}= \\
& g_{n} f_{n+1}
\end{aligned}
$$
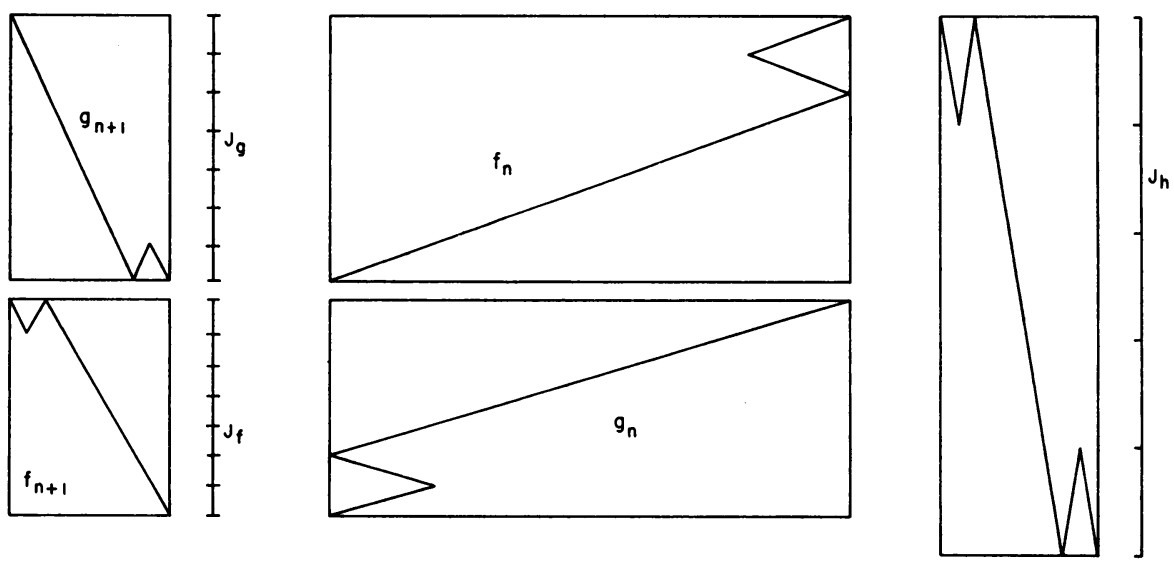

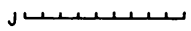

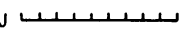

FIGURE 11

(2k)-set of $J$, and $f_{n+1}=f_{n}$ and $g_{n+1}=g_{n}$ on $J$, so case (a) is satisfied for both 6 and 7 . For case II, assume that $f_{n} / J_{g}$ is linear and $g_{n} / J_{f}$ is a hook function; then $S_{n} \cap J_{g}$ is the $(2 k)$-set of $J_{g}, g_{n} / J_{f}$ has order $2 k+2$, and $S_{n} \cap J_{f}$ is the $(2 k+2)$-set of $J_{f}$. 
We defined $S_{n+1} \cap J$ to be the $(2 k+2)$-set of $J$ and $f_{n+1}=f_{n}$ on $J$; so $f_{n+1}$ is linear from $J$ onto $J_{f}, S_{n+1} \cap J$ is the $(2 k+2)$-set of $J$, and $S_{n} \cap J_{f}$ is the $(2 k+2)$-set of $J_{f}$. Thus case (a) holds for $6 . g_{n+1} / J$ was defined to be a $(2 k+2)$-hook function from $J$ onto $J_{g}$, with $S_{n+1} \cap J$ being the $(2 k+2)$-set of $J$ and $S_{n} \cap J_{g}$ the ( $\left.2 k\right)$-set of $J_{g}$, so case (b) holds for 7. If on the other hand $g_{n} / J_{f}$ is linear instead of $f_{n} / J_{g}$, then case (a) holds for 7 and case (b) for 6 . In cases III and IV both $f_{n} / J_{g}$ and $g_{n} / J_{f}$ are $(2 k+2)$ hook functions, and $S_{n} \cap J_{g}$ and $S_{n} \cap J_{f}$ are $(2 k+2)$-sets. In case III we defined $f_{n+1} / J$ and $g_{n+1} / J$ to be linear, and $S_{n+1} \cap J$ to be the $(2 k+2)$-set of $J$, so case (a) holds for 6 and 7. In case IV both $f_{n+1} / J$ and $g_{n+1} / J$ are defined as $(2 k+4)$-hook functions and $S_{n+1} \cap J$ as the $(2 k+4)$-set of $J$, so case (b) holds for 6 and 7 . Thus requirements 6 and 7 of $L(n+1)$ are satisfied in all four cases.

For requirement 4 we note that in both cases (a) and (b) of 6 and 7 we have $f_{n+1}\left(S_{n+1} \cap J\right)=S_{n} \cap J_{f}, f_{n+1}$ linear on $(n+1)$-intervals, and $(n+1)$-intervals mapped onto (n)-intervals. Thus on all of $I, f_{n+1}\left(S_{n+1}\right) \subset S_{n}$ and $f_{n+1}$ maps $(n+1)$ intervals linearly onto $(n)$-intervals. Since $f_{n+1} / J$ was defined to agree in direction with $f_{n} / J, f_{n+1} / J$ agrees with $f_{n} / J$ on $S_{n} \cap J$, the end points of $J$; thus $f_{n+1}$ agrees with $f_{n}$ on $S_{n}$. For $i \leqq n$, by $L(n) f_{n}$ agrees with $f_{i}$ on $S_{i}$, and $S_{i} \subset S_{n}$, so $f_{n+1}$ agrees with $f_{i}$ on $S_{i}$. In all cases $f_{n+1}(J)=f_{n}(J)=J_{f}$, where $J$ is an $(n)$-interval. If $J_{i}$ is an $(i)$ interval and $i \leqq n$, then by $L(n) f_{n}\left(J_{i}\right)=f_{i}\left(J_{i}\right) ; J_{i}$ is the union of $(n)$-intervals, and the images of $(n)$-intervals under $f_{n+1}$ and $f_{n}$ coincide, so $f_{n+1}\left(J_{i}\right)=f_{n}\left(J_{i}\right)=f_{i}\left(J_{i}\right)$. Since $f_{i}\left(J_{i}\right)$ is an $(i-1)$-interval, for $x$ in $J_{i} f_{n+1}(x)$ and $f_{i}(x)$ lie in the same (i-1)-interval, so $\left|f_{n+1}(x)-f_{i}(x)\right| \leqq M_{i-1}$ and $\left\|f_{n+1}-f_{i}\right\| \leqq M_{i-1}$. Thus requirement 4 of $L(n+1)$ is satisfied. Requirement 5 is satisfied by exactly the same arguments for $g$ instead of $f$.

The only remaining requirement is number 3 , the "diagram commutativity," or showing that $f_{n+1}$ and $g_{n+1}$ as defined make the diagram in Figure 8 commutative. For case I all four functions are linear, so the two composites $f_{n} g_{n+1}$ and $g_{n} f_{n+1}$ are linear; but the composites agree on the end points of $J$, so they must agree on all of $J$.

For cases II and III the following observation is helpful. Let $A, B, C$, and $D$ be intervals, $V$ and $W$ linear onto functions, and $H$ a hook function from $B$ onto $C$, so that we have

$$
A \stackrel{V}{\longrightarrow} B \stackrel{H}{\longrightarrow} C \stackrel{W}{\longrightarrow} D .
$$

Then $H V$ and $W H$ are hook functions of the same order as $H, H V$ is the same type as $H$, and $W H$ is the same type as $H$ if and only if $W$ is sense-preserving (increasing).

Now for case II assume again that $f_{n} / J_{g}$ is linear. Then $f_{n+1} / J$ is linear, and $g_{n} / J_{f}$ and $g_{n+1} / J$ are hook functions of the same order. By the preceding remark $g_{n} f_{n+1}$ is a hook function of the same order and type as $g_{n} / J_{f}$, and $f_{n} g_{n+1}$ is a hook function of the same order as $g_{n} / J_{f}$. If $f_{n} / J_{g}$ is increasing, then $g_{n+1} / J$ is defined to be the same type as $g_{n} / J_{f}$, and by the remark $f_{n} g_{n+1}$ has the same type as $g_{n+1} / J$ which is the same as the type of $g_{n} / J_{f}$. If $f_{n} / J_{g}$ is decreasing, then $g_{n+1} / J$ is the opposite type as $g_{n} \mid J_{f}$, but $f_{n} g_{n+1}$ is the opposite type as $g_{n+1} / J$; so again $f_{n} g_{n+1}$ has the same type 
as $g_{n} / J_{f}$. Thus $f_{n} g_{n+1}$ and $g_{n} f_{n+1}$ are both hook functions of the same order and type as $g_{n} / J_{f}$. Since they agree on the end points of $J$, they also have the same direction. Order, type, and direction determine a hook function, so $f_{n} g_{n+1}$ and $g_{n} f_{n+1}$ must be the same function on $J$. By symmetry this conclusion is valid when it is $g_{n} / J_{f}$ which is the linear function.

For case III $f_{n} / J_{g}$ and $g_{n} / J_{f}$ are hook functions of the same order and type, and $f_{n+1} / J$ and $g_{n+1} / J$ are linear. Then $f_{n} g_{n+1}$ and $g_{n} f_{n+1}$ are hook functions of the same order and type as $f_{n} / J_{g}$ and $g_{n} / J_{f}$. Since they agree on the end points of $J$, they are hook functions of the same order, type, and direction; hence they are the same function and agree everywhere.

Case IV is more complicated than the other three. For each combination of directions of $f_{n} / J_{g}, g_{n} / J_{f}, f_{n} / J$, and $g_{n} / J$ and types of $f_{n} / J_{g}$ and $g_{n} / J_{f}$ the definition gives us a different set of four functions. This would appear to be six binary options, or $2^{6}=64$ different combinations, for a given value of $k$. But the fact that $f_{n} g_{n}$ and $g_{n} f_{n}$ agree on the end points of $J$, hence have the same direction, requires that of the four functions an even number must be increasing and an even number decreasing. Thus there are only three independent choices of direction. Let us assume these are $f_{n} / J_{g}, g_{n} / J_{f}$, and $f_{n} / J$, with the direction of $g_{n} / J$ depending on the other three. Recall that the directions of $f_{n+1} / J$ and $g_{n+1} / J$ were defined to be the same as $f_{n} / J$ and $g_{n} / J$. Now since for case IV $f_{n} / J_{g}$ and $g_{n} / J_{f}$ have opposite types, we know that one of them is maximum and the other minimum. Then the types of $f_{n+1} / J$ and $g_{n+1} / J$, according to the definition, are determined by the types and directions of $f_{n} / J_{g}$ and $g_{n} / J_{f}$. Thus the only free choices are whether $f_{n} / J_{g}$ is maximum or minimum, and the choice of the direction of three of the functions, a total of $2^{4}=16$ possible combinations.

If we observe that the problem is symmetric in $f$ and $g$, we can reduce the number of combinations that must be considered to eight, for without loss of generality we can assume that, say, $f_{n} / J_{g}$ is maximum and $g_{n} / J_{f}$ is minimum. When we make this assumption, the type of $f_{n+1} / J$ depends only on the direction of $g_{n} / J_{f}$, and the type of $g_{n+1} / J$ depends only on the direction of $f_{n} / J_{g}$. Thus we have the eight combinations given on the following table. Here the quantities in parentheses are those either fixed or dependent on other choices.

\begin{tabular}{|c|c|c|c|c|c|c|c|c|}
\hline & & & & & & & & \\
\hline & dir & (type) & dir & (type) & dir & (type) & (dir) & (type) \\
\hline 1. & incr & $\max$ & incr & $\min$ & incr & $\max$ & incr & $\min$ \\
\hline 2. & incr & $\max$ & incr & $\min$ & decr & $\max$ & decr & $\min$ \\
\hline 3. & incr & $\max$ & decr & $\min$ & incr & $\min$ & decr & $\min$ \\
\hline 4. & incr & $\max$ & decr & $\min$ & decr & $\min$ & incr & $\min$ \\
\hline 5. & decr & $\max$ & incr & $\min$ & incr & $\max$ & decr & $\max$ \\
\hline 6. & decr & $\max$ & incr & $\min$ & decr & $\max$ & incr & $\max$ \\
\hline 7. & decr & $\max$ & decr & $\min$ & incr & $\min$ & incr & $\max$ \\
\hline 8 & decr & $\max$ & decr & $\min$ & decr & $\min$ & decr & $\max$ \\
\hline
\end{tabular}


Rather than presenting the computations for all eight cases, which are similar, one will be worked out as an example. Case 2 is a good illustration since it includes all four kinds of hook functions. It is depicted in Figure 11 with $k=3$.

For the purpose of the proof we will denote the elements of $S_{n+1} \cap J$ as $\left\{a_{1}, a_{2}, \ldots, a_{2 k+4}\right\}$, the elements of $S_{n} \cap J_{f}$ as $\left\{b_{1}, b_{2}, \ldots, b_{2 k+2}\right\}$, the elements of $S_{n} \cap J_{g}$ as $\left\{c_{1}, \ldots, c_{2 k+2}\right\}$, and the elements of $S_{n-1} \cap J_{h}$ as $\left\{d_{1}, \ldots, d_{2 k}\right\}$. Then the four functions are defined as follows, observing that they are continuous and linear where not otherwise defined.

$$
\begin{aligned}
f_{n+1}\left(a_{1}\right) & =b_{2 k+2} ; \quad f_{n+1}\left(a_{2}\right)=b_{2 k+1} ; \quad f_{n+1}\left(a_{i}\right)=b_{2 k+5-i}, \quad i \geqq 3 . \\
g_{n+1}\left(a_{i}\right) & =c_{2 k+3-i}, \quad i \leqq 2 k+2 ; \quad g_{n+1}\left(a_{2 k+3}\right)=c_{2} ; g_{n+1}\left(a_{2 k+4}\right)=c_{1} . \\
f_{n}\left(c_{i}\right) & =d_{i}, \quad i \leqq 2 k ; \quad f_{n}\left(c_{2 k+1}\right)=d_{2 k-1} ; \quad f_{n}\left(c_{2 k+2}\right)=d_{2 k} . \\
g_{n}\left(b_{1}\right) & =d_{1} ; \quad g_{n}\left(b_{2}\right)=d_{2} ; \quad g_{n}\left(b_{i}\right)=d_{i-2}, \quad i \geqq 3 .
\end{aligned}
$$

Let us then check the commutativity. For $a_{1}$ and $a_{2}$ we have

$$
\begin{aligned}
& f_{n} g_{n+1}\left(a_{1}\right)=f_{n}\left(c_{2 k+2}\right)=d_{2 k} ; \quad g_{n} f_{n+1}\left(a_{1}\right)=g_{n}\left(b_{2 k+2}\right)=d_{2 k} . \\
& f_{n} g_{n+1}\left(a_{2}\right)=f_{n}\left(c_{2 k+1}\right)=d_{2 k-1} ; \quad g_{n} f_{n+1}\left(a_{2}\right)=g_{n}\left(b_{2 k+1}\right)=d_{2 k-1} .
\end{aligned}
$$

For $3 \leqq i \leqq 2 k+2$ we have

$$
f_{n} g_{n+1}\left(a_{i}\right)=f_{n}\left(c_{2 k+3-i}\right)=d_{2 k+3-i} ; \quad g_{n} f_{n+1}\left(a_{i}\right)=g_{n}\left(b_{2 k+5-i}\right)=d_{2 k+3-i} \text {. }
$$

And at the other end, for $a_{2 k+3}$ and $a_{2 k+4}$,

$$
\begin{array}{ll}
f_{n} g_{n+1}\left(a_{2 k+3}\right)=f_{n}\left(c_{2}\right)=d_{2} ; & g_{n} f_{n+1}\left(a_{2 k+3}\right)=g_{n}\left(b_{2}\right)=d_{2} . \\
f_{n} g_{n+1}\left(a_{2 k+4}\right)=f_{n}\left(c_{1}\right)=d_{1} ; & g_{n} f_{n+1}\left(a_{2 k+4}\right)=g_{n}\left(b_{1}\right)=d_{1} .
\end{array}
$$

Thus the diagram is commutative on $S_{n+1} \cap J$, and since the composites are linear in between, the diagram in Figure 8 must be commutative on all of $J$. Note that the composites are both a "double hook" function, which in this case is decreasing on $J$.

Similarly, in the other seven cases the diagram is commutative, so requirement 3 of $L(n+1)$ is satisfied in case IV. This completes the proof that $L(n)$ implies $L(n+1)$, and hence the proof of the lemma.

THEOREM. There exist continuous functions $f$ and $g$ which map the unit interval I onto itself and commute under functional composition but have no common fixed point.

Proof. From the lemma we have two sequences of functions $\left\{f_{i}\right\}$ and $\left\{g_{i}\right\}$ which satisfy requirements $1-7$. To prove the theorem it must be shown that the sequences converge uniformly to continuous functions $f$ and $g$, that $f$ and $g$ commute, and that $f$ and $g$ have no comon fixed point.

To prove uniform convergence of $\left\{f_{i}\right\}$ it must be shown that $\left\{f_{i}\right\}$ is a Cauchy sequence relative to the sup norm $\|\cdots\|$ on $I$. So let an $\varepsilon>0$ be chosen. There is an $N$ such that $(1 / 3)^{N-1}<\varepsilon$. Then when $n \geqq m>N$, by requirements 2 and 4 we have

$$
\left\|f_{n}-f_{m}\right\| \leqq M_{m-1} \leqq(1 / 3)^{m-1}<(1 / 3)^{N-1}<\varepsilon .
$$


Thus $\left\{f_{i}\right\}$ converges uniformly on $I$, so that $\left\{f_{i}\right\}$ has a unique continuous limit function $f$. Similarly $\left\{g_{i}\right\}$ converges uniformly to a continuous limit function $g$.

To get commutativity we use the inequality

$$
\begin{aligned}
|f g(x)-g f(x)| \leqq & \left|f(g(x))-f_{n}(g(x))\right|+\left|f_{n}(g(x))-f_{n}\left(g_{n+1}(x)\right)\right| \\
& +\left|f_{n} g_{n+1}(x)-g_{n} f_{n+1}(x)\right|+\left|g_{n}\left(f_{n+1}(x)\right)-g_{n}(f(x))\right| \\
& +\left|g_{n}(f(x))-g(f(x))\right| .
\end{aligned}
$$

Now let $\varepsilon>0$ be given. We can choose $N_{1}$ such that $n>N_{1}$ implies $\left\|f-f_{n}\right\|<\varepsilon / 4$; $N_{2}$ such that $n>N_{2}$ implies $\left\|g-g_{n}\right\|<\varepsilon / 4 ; N_{3}$ and $\delta_{3}$ such that $n, m>N_{3}$ and $|x-y|<\delta_{3}$ imply $\left|f_{n}(x)-f_{m}(y)\right|<\varepsilon / 4 ; N_{4}$ such that $n>N_{4}$ implies $\left\|g-g_{n}\right\|<\delta_{3}$; $N_{5}$ and $\delta_{5}$, and then $N_{6}$ defined for $g$ as $N_{3}, \delta_{3}$, and $N_{4}$ were for $f$. Then since $\left\|f_{n} g_{n+1}-g_{n} f_{n+1}\right\|=0$ for all $n$, when $n>\max \left(N_{1}, N_{2}, N_{3}, N_{4}, N_{5}, N_{6}\right)$ we have

$$
|f g(x)-g f(x)|<\varepsilon / 4+\varepsilon / 4+0+\varepsilon / 4+\varepsilon / 4=\varepsilon .
$$

Thus the limit functions commute.

For the final portion of the proof of the theorem it must be shown that $f$ and $g$ have no common fixed point. This will be done by using the characteristics of $f_{2}$ and $g_{2}$ and requirements 4 and 5 of the lemma. Referring back to Figure 3, we can see the manner in which $f_{2}$ and $g_{2}$ map (2)-intervals onto (1)-intervals. Now if $J_{2}$ is a (2)-interval and $f_{2}\left(J_{2}\right)=J_{1}$, a (1)-interval, by requirement 4 whenever $i \geqq 2$, we must have $f_{i}\left(J_{2}\right)=f_{2}\left(J_{2}\right)=J_{1}$. Thus in the limit, $f\left(J_{2}\right)=J_{1}$. This means that the graph of $f$ is in one of the rectangles of Figure 3 if and only if the graph of $f_{2}$ is. Comparing the rectangles through which the graph of $f_{2}$ passes with the rectangles through which the diagonal passes, it is clear that all fixed points of $f$ must lie in the third, seventh, and ninth (2)-intervals. In a similar manner, comparing the graph of $g_{2}$ with the rectangles and the diagonal shows that all the fixed points of $g$ must lie in the first, fifth, and eleventh (2)-intervals. Since no (2)-interval can contain fixed points of both $f$ and $g$, they can have no common fixed point.

\section{REFERENCES}

1. Glen Baxter, On fixed points of the composite of commuting functions, Proc. Amer. Math. Soc. 15 (1964), 851-855.

2. - personal communication, November 19, 1965.

3. Glen Baxter and J. T. Joichi, On permutations induced by commuting functions, and an imbedding question, Math. Scand. 13 (1963), 140-150.

4. - On functions that commute with full functions, Nieuw Arch. Wisk. (3) 12 (1964), 12-18.

5. - - On a class of commuting functions, Nieuw Arch. Wisk. (3) 12 (1964), 126-136.

6. H. D. Block and H. P. Thielman, Commutatice polynomials, Quart. J. Math. Oxford Ser. (2) 2 (1951), 241-243.

7. William M. Boyce, Generation of a class of permutations associated with commuting functions, Mathematical Algorithms 2 (1967), 19-26.

8. S. C. Chu and R. D. Moyer, On continuous functions, commuting functions, and fixed points, Fund. Math. 59 (1966), 91-95. 
9. Haskell Cohen, On fixed points of commuting functions, Proc. Amer. Math. Soc. 15 (1964), 293-296.

10. Ralph DeMarr, A common fixed point theorem for commuting mappings, Amer. Math. Monthly 70 (1963), 535-537.

11. Nelson Dunford and Jacob T. Schwartz, Linear Operators, Part I, Interscience, New York, 1958.

12. J. H. Folkman, On functions that commute with full functions, Proc. Amer. Math. Soc. 17 (1966), 383-386.

13. J. P. Huneke, Two counterexamples to a conjecture on commuting continuous functions of the closed unit interval, Abstract 67T-231, Notices Amer. Math. Soc. 14 (1967), 284.

14. - On common fixed points of commuting continuous functions on an interval, Doctoral Dissertation, Wesleyan Univ., Middletown, Conn., 1967.

15. J. R. Isbell, Commuting mappings of trees, Research Problem \#7, Bull. Amer. Math. Soc. 63 (1957), 419.

16. J. T. Joichi, On functions that commute with full functions and common fixed points, Nieuw Arch. Wisk. (3) 14 (1966), 247-251.

17. Gerald Jungck, Commuting mappings and common fixed points, Amer. Math. Monthly 73 (1966), 735-738.

18. J. E. Maxfield and W. J. Mourant, Common fixed points of commuting continuous functions on the unit interval, Nederl. Akad. Wetensch. Proc. Ser. A 68; Indag. Math. 27 (1965), 668-670.

19. J. F. Ritt, Permutable rational functions, Trans. Amer. Math. Soc. 25 (1923), 399-448.

20. A. J. Schwartz, Common periodic points of commuting functions, Michigan Math. J. 12 (1965), 353-355.

21. A. L. Shields, On fixed points of commuting analytic functions, Proc. Amer. Math. Soc. 15 (1964), 703-706.

Bell Telephone Laboratories, InC., Murray Hill, New Jersey

TUlane UNIVERSITY, New OrLeans, Louisiana 